\title{
Numerical simulation of the flow field and concentration distribution in the bulk growth of silicon carbide crystals
}

\author{
Jing Lu, Zi-Bing Zhang, Qi-Sheng Chen* \\ Institute of Mechanics, Chinese Academy of Sciences, 15 Bei Si Huan Xi Road, Beijing 100080, PR China
}

Available online 12 June 2006

\begin{abstract}
The physical vapor transport (PVT) method is being widely used to grow large-size single SiC crystals. The growth process is associated with heat and mass transport in the growth chamber, chemical reactions among multiple species as well as phase change at the crystal/gas interface. The current paper aims at studying and verifying the transport mechanism and growth kinetics model by demonstrating the flow field and species concentration distribution in the growth system.

We have developed a coupled model, which takes into account the mass transport and growth kinetics. Numerical simulation is carried out by employing an in-house developed software based on finite volume method. The results calculated are in good agreement with the experimental observation.
\end{abstract}

(C) 2006 Elsevier B.V. All rights reserved.

PACS: $47.70 . \mathrm{Nd} ; 51.10 .+\mathrm{y} ; 81.10 . \mathrm{Aj}$

Keywords: A1. Computer simulation; A1. Growth model; A1. Mass transfer; A2. Growth from vapor; A2. Seed crystals; B2. Semiconducting silicon compounds

\section{Introduction}

Wide-bandgap silicon carbide $(\mathrm{SiC})$ is a promising semiconductor material for electronic and opto-electronic devices involving high power, high temperature, high frequency and intense radiation. $\mathrm{SiC}$ based device technology as well as the volume production of nitride based high brightness blue and green LEDs fabricated on $\mathrm{SiC}$ substrates have made tremendous progress within the last several years [1].

Large size bulk SiC crystals are commonly grown by the physical vapor transport (PVT) method [2-8]. The bulk growth process is associated with many important physical phenomena, such as electromagnetic field, radio frequency (RF) induction heating, conduction and radiation heat transfer, sublimation and condensation, mass transfer and so on. The species transport process involves sublimation of a heated $\mathrm{SiC}$ powder charge in the bottom region of a graphite crucible, transport of sublimated vapor through

\footnotetext{
*Corresponding author. Tel.: 861062564199 ; fax: + 861062615524 .

E-mail address: qschen@imech.ac.cn (Q.-S. Chen).
}

an inert gas environment, and condensation on a cold preoriented seed mounted at the bottom of the lid of the crucible.

Process modeling in various degrees of complexity has been performed by many investigators to achieve better understanding of the transport mechanism. Bloem and Giling [9] formulated some simple growth kinetics for the semiconductor CVD processes. Kaneko [10,11] compiled several growth models for various kinds of PVD and CVD processes, considering the impinging molecular flux of a rate-determining species in equilibrium, and used a sticking coefficient to relate the growth rate with equilibrium vapor pressure of the species. Balkas et al. [14] pointed out that the growth rate is not only related to the growth temperature but also to the temperature gradient. Chen and co-workers [12,13] proposed a kinetics model for $\mathrm{SiC}$ vapor growth, assuming that the species transport rate near the seed is proportional to the supersaturation of $\mathrm{SiC}_{2}$ and $\mathrm{Si}$ vapor, and obtained the growth rate profile across the seed surface from the temperature distribution in a $3^{\prime \prime}$ growth system. In the present paper, we solved the incompressible Navier-Stokes equations and species 
equation coupled with the growth kinetics model to obtain the flow field and species concentration distribution in the growth system.

\section{Physical and mathematical model}

The SiC PVT growth system consists of an RF induction coil, a graphite susceptor and insulation as shown in Fig. 1. A sintered $\mathrm{SiC}$ powder charge is placed inside the cylindrical graphite crucible. A seed crystal is attached to the bottom of the lid of the crucible. The crucible is heated to approximately $2100-2800 \mathrm{~K}$ in an argon environment by $\mathrm{RF}$ heating leading to dissolving and sublimation of the charge.

The $\mathrm{SiC}$ vapor species are transported from the charge to the seed by the advection (also called Stefan flow) due to volumetric sublimation of $\mathrm{SiC}$ charge and the Fickian diffusion driven by concentration gradient. An axisymmetric coordinate system is introduced where the axial coordinate $x$ is set as 0 (zero) at the charge and as $L$ at the seed (Fig. 1). Navier-Stokes equations are employed here to simulate the flow of the gas mixture and a species equation is used to describe the species transport. The governing equations for flow and species transport of the gas mixture can be written as

$\nabla \cdot(\bar{\rho} \mathbf{v})=0$,

$\frac{\partial \bar{\rho} u}{\partial t}+\nabla \cdot(\bar{\rho} u \mathbf{v})=-\frac{\partial p}{\partial x}+\mu \nabla^{2} u+\bar{\rho} \alpha\left(T-T_{\text {bot }}\right) g$

$\frac{\partial \bar{\rho} v}{\partial t}+\nabla \cdot(\bar{\rho} v \mathbf{v})=-\frac{\partial p}{\partial r}+\mu \nabla^{2} v-\mu \frac{v}{r^{2}}$ $\frac{\partial c_{\mathrm{A}}}{\partial t}+\nabla \cdot\left(c_{\mathrm{A}} \mathbf{v}\right)=D_{\mathrm{AB}} \nabla^{2} c_{\mathrm{A}}$,

where $\bar{\rho}$ is the density of the gas mixture, $\mathbf{v}=(u, v)$ is velocity vector, $u$ is axial velocity component, $v$ is radial velocity component, $c$ is the molar concentration, $\mu$ is the dynamic viscosity, $\alpha$ is thermal expansion coefficient, $T$ is local temperature, $T_{\mathrm{bot}}$ is the reference temperature at the bottom of the crucible, $g$ is gravitational acceleration, and subscripts $\mathrm{A}$ and $\mathrm{B}$ denote rate-determining species and inert gas, respectively, $D_{\mathrm{AB}}$ is binary diffusion coefficient.

A growth kinetics model has been developed by Chen et al. [12] which assumes that the species transport rate near the seed is proportional to supersaturation of the ratedetermining species, A, such that,

$J_{\mathrm{A}}=\chi_{\mathrm{A}}\left(p_{\mathrm{A}}(L)-p_{\mathrm{A}}^{*}(L)\right)$,

where $J_{\mathrm{A}}$ is the molar flux of the vapor species $\mathrm{A}$, the superscript $*$ denotes equilibrium state, $p_{\mathrm{A}}$ and $p_{\mathrm{A}} *$ is the vapor pressure and equilibrium vapor pressure of the transport species, respectively, $\chi_{\mathrm{A}}=\left(1 / \sqrt{2 \pi M_{\mathrm{SiC}} R T}\right), R$ is the gas constant, $M_{\mathrm{SiC}}$ and is the molecular weight of $\mathrm{SiC}$.

Boundary conditions at the seed and charge surface for the governing equations take the form

$J_{\mathrm{A}}=\left.\left(u c_{\mathrm{A}}-D_{\mathrm{AB}} \frac{\partial c_{\mathrm{A}}}{\partial x}\right)\right|_{x=L}$,

$J_{\mathrm{B}}=\left.\left(u c_{\mathrm{B}}-D_{\mathrm{BA}} \frac{\partial c_{\mathrm{B}}}{\partial x}\right)\right|_{x=L}=0$,

$\left.p_{\mathrm{A}}\right|_{x=0}=p_{\mathrm{A}}^{*}\left(T_{\max }\right)$,

$J_{\mathrm{B}}=\left.\left(u c_{\mathrm{B}}-D_{\mathrm{BA}} \frac{\partial c_{\mathrm{B}}}{\partial x}\right)\right|_{x=0}=0$.

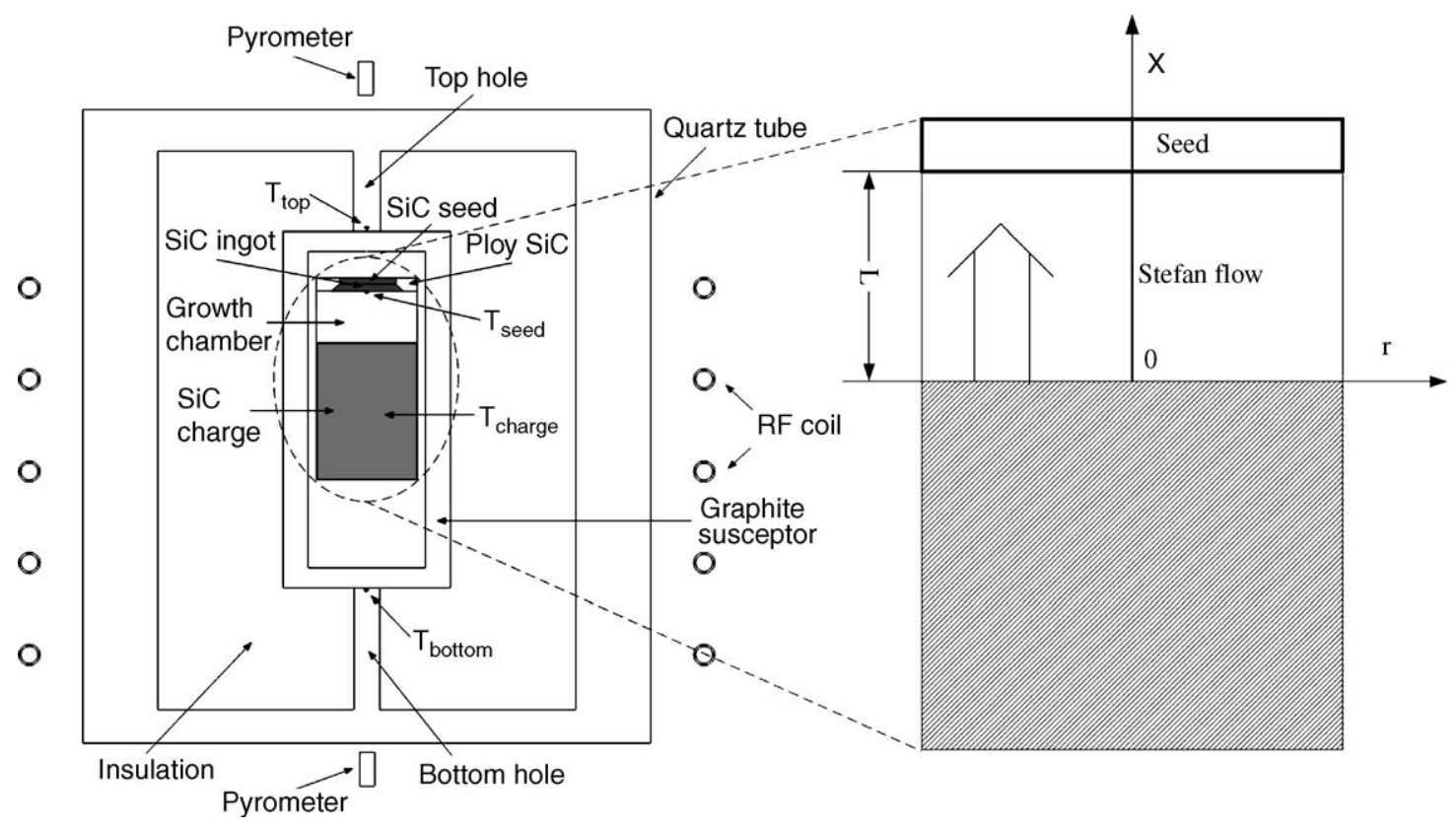

Fig. 1. Growth system (left) and crucible (right). 
The above flux boundary conditions couple the species transport equation and the growth kinetics at the seed.

\section{Results and discussion}

Computations on species distribution and flow field are performed by a finite-volume-based code. Temperature field inside the growth system as shown in Fig. 2a was obtained using the procedures as in Ref. [13]. Current in the induction coil is chosen as $1400 \mathrm{~A}$ and the induction frequency is chosen as $8 \mathrm{kHz}$ in this case. The maximum temperature in the charge is $2792 \mathrm{~K}$ appearing in the region near the crucible wall at the bottom of the charge. Temperature at the charge is $2695 \mathrm{~K}$ on average and is higher than that of the seed, say, $2665 \mathrm{~K}$ on average. The temperature difference between the charge and seed forms a temperature gradient, which is critical for the transport of $\mathrm{SiC}$ species and the enlargement of the bulk crystal. By solving the governing Eqs. (1)-(4) combined with the corresponding boundary conditions, the flow field in the crucible can be obtained based on the calculated temperature field as is shown in Fig. 2b. Total pressure in the
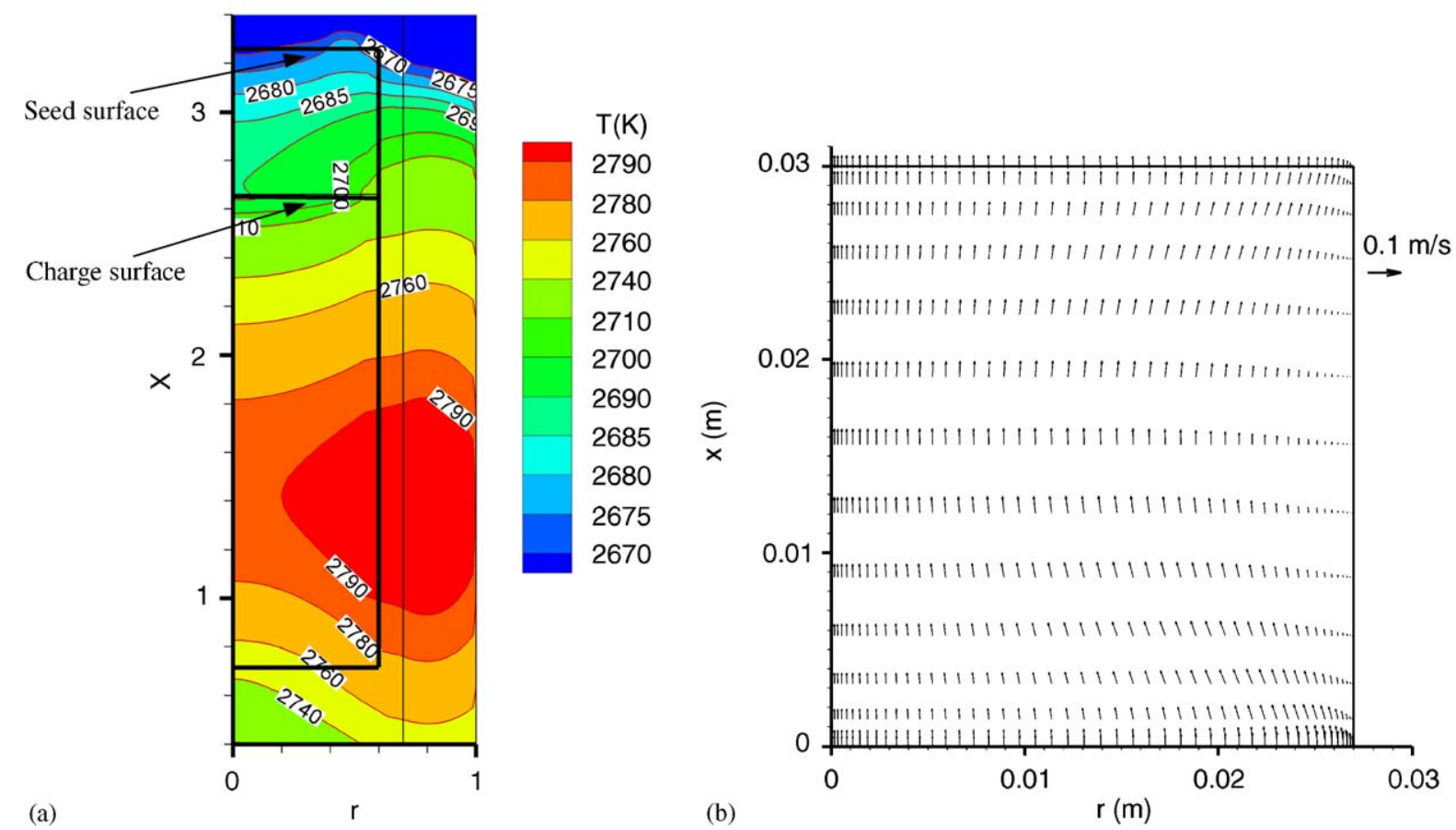

Fig. 2. (a) Temperature field in the crucible and (b) flow field between charge and seed.
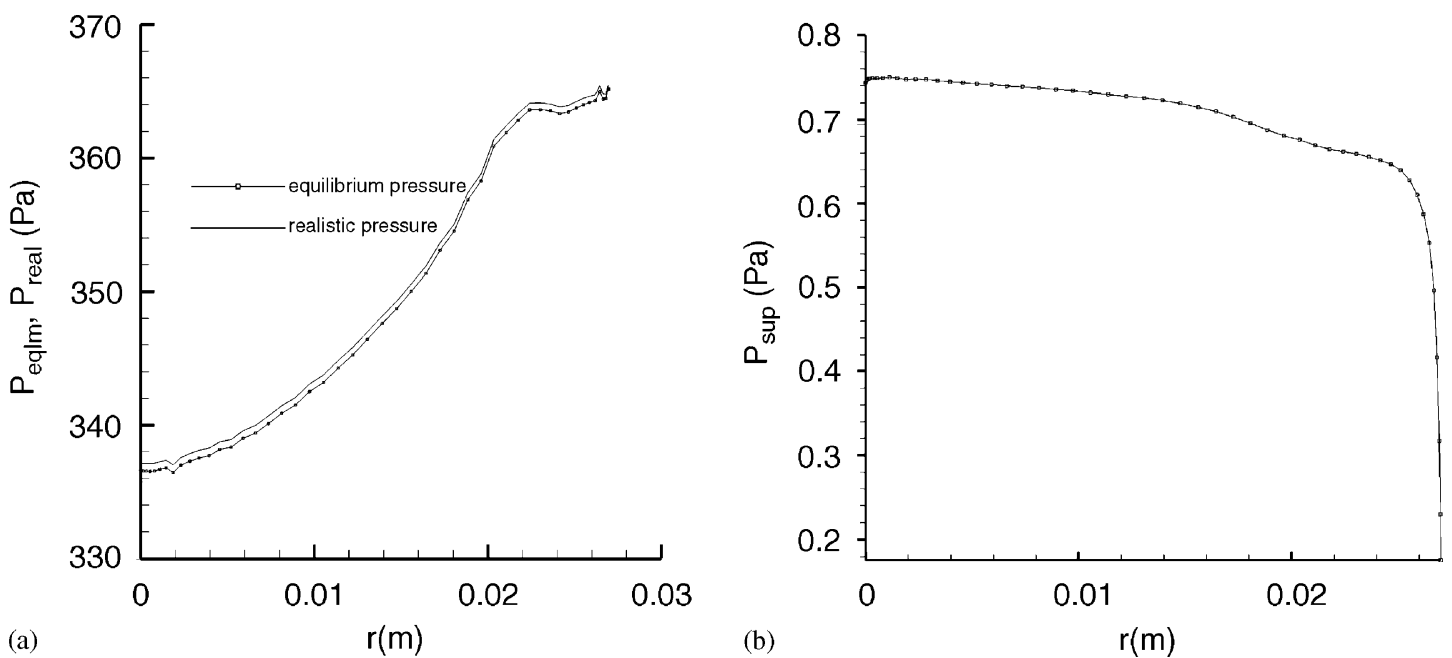

Fig. 3. (a) Equilibrium pressure and actual vapor pressure of $\mathrm{SiC}$ vapor species and (b) supersaturation of $\mathrm{SiC}$ vapor species. 
growth system is controlled at $8 \mathrm{kPa}$. The magnitude of the velocity is of the order of $0.1 \mathrm{~m} / \mathrm{s}$. At the charge surface, velocity is larger near the wall than that in the center.

The species equation is solved to obtain the concentration distribution on the seed surface as shown in Fig. 3a. Distributions of equilibrium pressure and actual vapor pressure of the $\mathrm{SiC}$ vapor species are shown in Fig. 3a. It can be seen that both of the pressures increase from the center to the edge of the seed. The magnitude varies from 338 to $366 \mathrm{~Pa}$. Realistic pressure is a little higher than the equilibrium pressure so as to maintain a supersaturation at the seed. Fig. $3 b$ shows the distribution of supersaturation along the seed. Though the difference is only several $\mathrm{Pa}$, it is critical for the transport of the $\mathrm{SiC}$ vapor species to the seed. It is shown that the value is larger at the center than that at the edge. Since the growth rate is proportional to the supersaturation of $\mathrm{SiC}$ vapor species, the growth rate is larger at the center than near the wall leading to the convex shape of bulk crystal. This has been verified by experimental observations that the as-grown $\mathrm{SiC}$ single crystal surface is convex in shape.

\section{Conclusions}

Numerical simulation has proved to be an accurate and efficient method to obtain a better understanding of the transport mechanisms in the growth process. The Navier-Stokes equations and species equation were used to obtain the flow field and species distribution. Mass transport and growth kinetics are coupled by imposing flux boundary conditions. By setting up an axi-symmetric model for the flow and species transport of the gas mixture, the flow field and concentration distribution are studied. Numerical results are in good agreement with the experimental observations. The present model is able to capture the characteristics of the mass transport and growth kinetics in the $\mathrm{SiC}$ growth process by the PVT method, thus indicating that we can optimize the growth process by the modeling and simulation approach.

\section{Acknowledgments}

This work has been supported by the National Science Foundation of China (10472126, 10432060) and the Knowledge Innovation Program of the Chinese Academy of Sciences.

\section{References}

[1] St.G. Müller, C.H. Carter, V.F. Tsvetkov, J. Crystal Growth 211 (2000) 325.

[2] Yu.M. Tairov, V.F. Tsvetkov, J. Crystal Growth 43 (1978) 209.

[3] Yu.M. Tairov, V.F. Tsvetkov, J. Crystal Growth 52 (1981) 146.

[4] D.L. Barrett, J.P. McHugh, H.M. Hobgood, R.H. Hopkins, P.G. McMullin, B.C. Clarke, W.J. Choyke, J. Crystal Growth 128 (1993) 358.

[5] G. Augustine, H.McD. Hobgood, V. Balakrishna, G. Dunne, R.H. Hopkins, Phys. Status Solidi B 202 (1997) 137.

[6] R.C. Glass, D. Henshall, V.F. Tsvetkov, C.H. Carter Jr., Phys. Status Solidi B 202 (1997) 149.

[7] A.R. Powell, S. Wang, G. Fechko, G.R. Brandes, Mater. Sci. Forum 264-268 (1998) 13.

[8] D. Hofmann, M. Bickermann, R. Eckstein, St.G. Muller, E. Schmitt, A. Weber, A. Winnacker, J. Crystal Growth 198/199 (1999) 1005.

[9] J. Bloem, L.J. Giling, Mechanisms of the chemical vapor deposition of silicon, in: E. Kaldis (Ed.), Current Topics in Materials Science, vol. 1, North-Holland, Amsterdam, 1978, pp. 147-342.

[10] T. Kaneko, J. Crystal Growth 69 (1984) 1.

[11] T. Kaneko, J. Crystal Growth 128 (1993) 354.

[12] Q.-S. Chen, H. Zhang, V. Prasad, C.M. Balkas, N.K. Yushin, S. Wang, J. Crystal Growth 224 (2001) 101.

[13] Q.-S. Chen, H. Zhang, V. Prasad, C.M. Balkas, N.K. Yushin, J. Heat Transfer 123 (2001) 1098.

[14] C.M. Balkas, A.A. Maltsev, M.D. Roth, N.K. Yushin, Mater. Sci. Forum 338-342 (2000) 79. 